\title{
New Upper Bounds for the Size of Permutation Codes via Linear Programming
}

\author{
Mathieu Bogaerts \\ Université Libre de Bruxelles \\ Service de Mathématiques, Faculté des Sciences Appliquées \\ CP 165/11 avenue Roosevelt 50 \\ B-1050 Brussels, Belgium \\ mbogaert@ulb.ac.be
}

Submitted: Jan 2, 2010; Accepted: Sep 30, 2010; Published: Oct 15, 2010

Mathematics Subject Classification: 05B15

\begin{abstract}
An $(n, d)$-permutation code of size $s$ is a subset $C$ of $S_{n}$ with $s$ elements such that the Hamming distance $d_{H}$ between any two distinct elements of $C$ is at least equal to $d$. In this paper, we give new upper bounds for the maximal size $\mu(n, d)$ of an $(n, d)$-permutation code of degree $n$ with $11 \leqslant n \leqslant 14$. In order to obtain these bounds, we use the structure of association scheme of the permutation group $S_{n}$ and the irreducible characters of $S_{n}$. The upper bounds for $\mu(n, d)$ are determined solving an optimization problem with linear inequalities.
\end{abstract}

\section{Permutation arrays and permutation codes}

An $(n, d)$-permutation code of distance $d$, size $s$ and degree $n$ is a non-empty subset $C$ of the symmetric group $S_{n}$ acting on the set $\{1, \ldots, n\}$ such that the Hamming distance between any two distinct elements of $C$ is at least equal to $d$. The Hamming distance between two permutations $\phi, \psi \in S_{n}$ is defined as $d_{H}(\phi, \psi)=|\{i \in\{1, \ldots, n\}: \phi(i) \neq \psi(i)\}|$. The weight of a permutation $\phi \in S_{n}$ if the number of non fixed points of $\phi$.

The $s \times n$ array $A$ associated to a $(n, d)$-permutation code $C=\left\{\phi_{1}, \ldots, \phi_{s}\right\}$ of size $s$ by $A_{i j}=\phi_{i}(j)$ has the following properties: every symbol 1 to $n$ occurs exactly in one cell of any row and any two rows disagree in at least $d$ columns. Such an array is called a permutation array (PA) of distance $d$, size $s$ and degree $n$.

Permutation codes have first been proposed by Ian Blake in 1974 as error-correcting codes for powerline communications [3]. This application motivates the study of the largest possible size that a permutation code can have. Upper bounds for the maximal size $\mu(n, d)$ of a permutation code with fixed parameters $n$ and $d$ have been studied by 
many authors, see e.g. Deza and Frankl [10], Cameron [6], and more intensively since Chu, Colbourn and Dukes [8], Tarnanen [15], and Han Vinck [2, 16]. An $(n, d)$ - permutation code $C$ of weight $w$ is an $(n, d)$ - permutation code such that all permutations have weight $w$. The maximal size of such a permutation code is denoted by $\mu(n, d, w)$.

An $(n, d)$-permutation code $C$ of size $s$ is maximal if $C$ is not contained in an $(n, d)$ permutation code of larger size $s^{\prime}>s$. Note that an $(n, d)$-permutation code reaching the maximal size $\mu(n, d)$ is necessarily maximal while the converse is not true. The most basic upper bounds on $\mu(n, d)$ appears in Deza and Frankl [10]:

Theorem 1. For $n \geqslant 3$ and $d \leqslant n$,

$$
\mu(n, d) \leqslant n \mu(n-1, d)
$$

and therefore

$$
\mu(n, d) \leqslant \frac{n !}{(d-1) !}
$$

In this paper, we will establish new bounds for $\mu(n, d)$ for small values of the parameters $n$ and $d$. In [15], H. Tarnanen uses the conjugacy scheme of the group $S_{n}$ in order to obtain new upper bounds for the size of a permutation code. We use this method to obtain new upper bounds for $\mu(n, d)$.

\section{Isometries}

A distance $D$ on $S_{n}$ is called left-invariant (resp. right-invariant) if $D(\phi, \psi)=D(\alpha \phi, \alpha \psi)$ (resp. $D(\phi, \psi)=D(\phi \alpha, \psi \alpha)$ ) for all $\alpha, \phi, \psi \in S_{n}$. A distance that is both left- and rightinvariant is said to be bi-invariant. For any bi-invariant distance, the left multiplications $l_{\alpha}: \phi \mapsto \alpha \phi$ and the right multiplications $r_{\alpha}: \phi \mapsto \phi \alpha^{-1}$ are isometries. As noticed by Deza and Huang [11], any bi-invariant distance is invertible: $D(\phi, \psi)=D\left(\phi^{-1}, \psi^{-1}\right)$, or equivalently, the inversion $i$, mapping each permutation onto its inverse, is an isometry. Let $\mathcal{R}$ (resp. $\mathcal{L}$ ) denote the group of all right (resp. left-) multiplications and $\mathcal{I}$ denote the group generated by the inversion $i$. We will say that the distance $D$ distinguishes the transpositions if there exists a constant $c$ such that $D(\phi, \psi)=c \Leftrightarrow \phi \psi^{-1}$ is a transposition. In 1960, Farahat characterized the isometry group $I s o(n)$ of the metric space $\left(S_{n}, d_{H}\right)$ [12]. Since the Hamming distance is bi-invariant and distinguishes the transpositions, the following result appears in [4] and generalizes the characterisation given by Farahat:

Theorem 2. Let $D$ be a bi-invariant distance distinguishing the transpositions on $S_{n}$

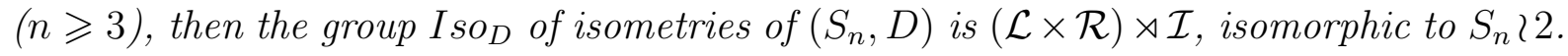

Every isometry $t \in I$ so $(n)$ can be uniquely written as $l_{\alpha} r_{\beta} i^{k}$ with $k=0$ or $1, \alpha, \beta \in S_{n}$. The action of a left multiplication $l_{\alpha}$ on a given code corresponds to the permutation under $\alpha$ of the symbols appearing in the PA associated to the code, and the action of a rightmultiplication $r_{\beta}$ is equivalent to the permutation under $\beta$ of the columns of the PA. In other words, classifying permutation codes up to isometry is equivalent to classifying PA's 
up to permutation of their rows, their columns, their symbols and up to the inversion. It immediately follows from this theorem that the autormorphism group of the conjugacy scheme of $S_{n}$ is precisely the isometry group of the metric space $\left(S_{n}, d_{H}\right)$.

\section{$3 \quad$ Linear programming bound}

A symmetric association scheme with $m$ classes is a finite set $X$ with $m+1$ relations $R_{0}, R_{1}, \ldots R_{m}$ on $X$ such that:

- $\left\{R_{0}, R_{1}, \ldots R_{m}\right\}$ is a partition of $X \times X$

- $R_{0}=\{(x, x) \mid x \in X\}$

- If $(x, y) \in R_{i}$, then $(y, x) \in R_{i}$ for all $x, y \in X$ and for all $i=0, \ldots, m$

- For each pair $(x, y) \in R_{k}$, the number $p_{i j}^{k}$ of elements $z \in X$ such that $(x, z) \in R_{i}$ and $(y, z) \in R_{j}$ only depends on $i, j$ and $k$

The numbers $p_{i j}^{k}$ are called intersection numbers of the association scheme. Let $n$ denote the size of the set $X$ and $n_{i}:=p_{i i}^{0} \quad i=0, \ldots, m$. The intersection matrices $L_{0}, \ldots, L_{m}$ are defined by: $\left(L_{i}\right)_{j k}=p_{i j}^{k}$. The relations $R_{i}$ can be described by their adjacency matrix $A_{i}$ : The adjacency matrix $A_{i}$ of the relation $R_{i}$ is the $n \times n$-matrix such that:

$$
\left(A_{i}\right)_{x y}= \begin{cases}1 & \text { if }(x, y) \in R_{i} \\ 0 & \text { otherwise }\end{cases}
$$

In terms of adjacency matrices the conditions defining the association scheme become:

- $\sum_{i=0}^{m} A_{i}=J$ where $J$ is the full one matrix, i.e. $J_{i j}=1$ for all $i, j$.

- $A_{0}=I$ where $I$ is the identity matrix,

- $A_{i}=A_{i}^{T}$ for all $i \in\{0, \ldots, m\}$

- $A_{i} A_{j}=\sum_{k=0}^{m} p_{i j}^{k} A_{k}$ for all $i, j \in\{0, \ldots, m\}$

The adjacency matrices commute and generate the commutative Bose Mesner algebra $\mathcal{A}$ of dimension $m+1$. The algebra $\mathcal{A}$ has a basis $E_{0}, \ldots, E_{m}$ such that:

1. $E_{i} E_{j}=\delta_{i j} E_{i}$

2. $\sum_{i=0}^{m} E_{i}=I$ 
The matrix $E_{0}$ can be taken as to be $\frac{J}{n}$ where $J$ is the full one matrix, i.e. $J_{i j}=1$ for all $i, j$. Let $P$ and $\frac{1}{n} Q$ be the basis transition matrices in $\mathcal{A}$ :

$$
\begin{gathered}
A_{j}=\sum_{i=0}^{m} P_{i j} E_{j} \\
E_{j}=\frac{1}{n} \sum_{i=0}^{m} Q_{i j} A_{j}
\end{gathered}
$$

We then obtain $P Q=Q P=n I$ and $A_{j} E_{i}=P_{i j} E_{i}$ The numbers $P_{i j}$ are the eigenvalues of $A_{j}$ with the columns of $E_{i}$ as corresponding eigenvectors.

Let $Y$ be a subset of $X$ and denote by $\chi$ the characteristic vector of $Y: \chi_{i}=1$ if $i \in Y$ and $\chi_{i}=0$ if $i \notin Y$. The inner distribution of a subset $Y$ of an association scheme is the vector

$$
\bar{a}=\left(a_{0}, \ldots a_{m}\right)
$$

where $a_{i}=\frac{1}{|Y|} \chi^{T} A_{i} \chi$. It is obvious that $a_{0}=1$ (because $A_{0}=I$ ) and $\sum_{i=0}^{m} a_{i}=|Y|$. For all $i=0, \ldots, m, a_{i}$ corresponds to the number of ordered pairs $(x, y) \in Y \times$ such that $(x, y) \in R_{i}$, divided by $|Y|$.

Theorem 3 (Delsarte [9],Th. 3.3, p. 26). The inner distribution $\bar{a}$ of a non empty set $Y$ of an association scheme satisfies $\bar{a} Q \geqslant 0$.

Let $Y$ be a subset of an association scheme such that $\forall x, y \in Y,(x, y) \notin R_{i}$ for all $i \in\{1, \ldots \delta-1\}$, or equivalently $(x, y) \in R_{i} \Rightarrow i=0$ or $\delta \leqslant i \leqslant m$. The inner distribution vector $\bar{a}$ of $Y$ satisfies:

$$
\left\{\begin{array}{l}
a_{0}=1 \\
a_{k}=0 \text { if } 1 \leqslant k \leqslant \delta-1 \\
a_{k} \geqslant 0 \text { if } \delta \leqslant k \leqslant m \\
\bar{a} Q \geqslant 0 \\
a_{0}+\sum_{i=\delta}^{m} a_{i}=|Y|
\end{array}\right.
$$

Theorem 4 (Delsarte [9], Th. 3.8,p.31).

Consider $a_{\delta}, \ldots, a_{m}$ as real variables and define $a^{*}=1+\sum_{i=\delta}^{m} a_{i}$ as the maximal value of this sum such that

$$
\left\{\begin{array}{l}
Q_{1 j}+\sum_{i=\delta}^{m} a_{i} Q_{i j} \geqslant 0 \quad j=0, \ldots, m \\
a_{i} \geqslant 0, \quad i=\delta, \ldots, m
\end{array}\right.
$$

Then $|Y| \leqslant a^{*}$. 


\section{Conjugacy scheme}

Any group $G$ defines a symmetric association scheme on its elements with relations defined by the conjugacy classes $C_{i}$ of $G$ for $\phi, \psi \in G,(\phi, \psi) \in R_{i} \Leftrightarrow \phi \psi^{-1} \in C_{i}$. For $G=S_{n}$, denote by $p(n)$ the number of conjugacy classes of $G$.

Let $\chi_{0}, \ldots, \chi_{m}$ be the irreducible characters of $S_{n}$, indexed in such a manner that $\chi_{0}(\alpha)=$ $1 \forall \alpha \in S_{n}$. There are $p(n)=m+1$ irreducible characters, where $p(n)$ is the number of conjugacy classes of $S_{n}$. Recall that the values of $\chi_{k}$ are integers, that the functions $\chi_{k}$ are constant on each conjugacy class and that $\sum_{k=0}^{m} \chi_{k}^{2}(I d)=n !$. The irreducible characters form an orthonormal basis of the set $C f\left(S_{n}\right)$ of class functions of $S_{n}$, for the product $<\cdot, \cdot>_{n}: C f^{2}\left(S_{n}\right) \rightarrow \mathbb{R}$ defined by

$$
<f, g>_{n}=\sum_{\alpha \in S_{n}} \frac{f(\alpha) g(\alpha)}{n !}
$$

Theorem 5 (Tarnanen, [15]). For the conjugacy scheme $\left(S_{n}, R_{0}, \ldots, R_{m}\right)$, the transition coefficents $Q_{i j}$ are given by:

$$
Q_{i j}=\chi_{j}(I d) \cdot \chi_{j}\left(C_{i}\right)
$$

Every $(n, d)$-permutation code $C$ is a subset of the conjugacy scheme. Suppose that the permutations of $S_{n}$ are indexed $\phi_{1}, \ldots, \phi_{n !}$. To avoid confusion, we will denote by $\xi_{C}$ the caracteristic vector of the code $C$, defined as $\left(\xi_{C}\right)_{i}=1$ if $\phi_{i} \in C$ and $\left(\xi_{C}\right)_{i}=0$ otherwise. For any $(n, d)$-permutation code $C$, the numbers $a_{i}=\xi_{C} A_{i} \xi_{C}^{T}$ are invariant under the action of $I s o(n)$ (see [4] for more information on invariants).

Theorem 6 (LP bound for permutation codes (Tarnanen,[15])). Let $D$ be a subset of $\{1, \ldots, m\}$ and $E$ any subset of $S_{n}$ such that for any distinct permutations $\phi, \psi,(\phi, \psi) \in$ $R_{i}$ with $i \in D$

Considering $a_{k}, \quad k \in D$ as real variables and denoting by $a^{*}$ the number $1+\sum_{i \in D} a_{i}$, the maximal value of this sum with

$$
\left\{\begin{array}{l}
\chi_{j}\left(C_{0}\right)+\sum_{i \in D} a_{i} \chi_{j}\left(C_{k}\right) \geqslant 0 \quad \forall j \in\{0, \ldots, m\} \\
a_{i} \geqslant 0, \quad i \in D
\end{array}\right.
$$

Then $|E| \leqslant a^{*}$.

If $D$ is a subset of indices of conjugacy classes whose elements have less than $n-d$ fixed points, this bound provides an upper bound for the size of a permutation code of distance $d$. The permutation characters of $S_{n}$ are available on programs as Magma [5] or GAP [13]. Using the "linprog" routine of Matlab [14], we obtain the bounds in Table 1. Note that the linear programming provides the values of the coefficients $a_{i}$, considered as real variables. On the other hand, if there exists an $(n, d)$ - permutation code $C$ whose size reaches the upper bound $a^{*}$ then the the numbers $b_{i}=a_{i} a^{*}=\xi_{C}^{T} A_{i} \xi_{C}$ are integers. 
The linear inequalities in theorem 6 lead to the following check routine of the feasability of the upper bound $a^{*}$. Let $d \leqslant n$ be fixed, and suppose that $a^{*}$ is the value obtained by linear programming bound of Theorem 6 . Then consider $b_{k}, \quad k \in D$ as integer variables and denote by $b^{*}$ the maximal value $1+\max \sum_{i \in D} b_{i}$, with

$$
\left\{\begin{array}{l}
a^{*} \chi_{j}\left(C_{0}\right)+\sum_{i \in D} b_{i} \chi_{j}\left(C_{i}\right) \geqslant 0 \quad \forall j \in\{0, \ldots, m\} \\
b_{i} \geqslant 0, \quad i \in D
\end{array}\right.
$$

Then the bound $a^{*}$ is feasable if $b^{*}=a^{* 2}$. The integer linear programming problem above can be solved using appropriate matlab routine [14].

\begin{tabular}{|l|c|c|}
\hline & LP bound & Previous known bound \\
\hline$\mu(13,4)$ & 367270674 & 479001600 \\
$\mu(11,5)$ & 362880 & 712800 \\
$\mu(12,5)$ & 6141046 & 7149277 \\
$\mu(13,5)$ & 75789398 & 78823048 \\
$\mu(11,6)$ & 138600 & 273402 \\
$\mu(12,6)$ & 1766160 & 3926242 \\
$\mu(13,6)$ & 21621600 & 29511947 \\
$\mu(11,7)$ & 32874 & 55440 \\
$\mu(12,7)$ & 361396 & 665280 \\
$\mu(13,7)$ & 4163390 & 8648640 \\
$\mu(13,8)$ & 879493 & 1235520 \\
\hline
\end{tabular}

Table 1: LP bound for $11 \leqslant n \leqslant 13$

Applying theorem 1 to the results of Table 1, we obtain recursive consequences. This leads to the upper bounds appearing in Table 2. The previous known bounds are due to Deza and Frankl [10].

As noticed by H. Tarnanen [15], many of the upper bounds obtained by linear programming coincide with the bound $\mu(n, d) \leqslant \frac{n !}{(d-1) !}$ of theorem 1 . For $14 \leqslant n \leqslant 16$, computations of the LP bound give $\frac{n !}{(d-1) !} \leqslant a^{*}$ for all $d \leqslant n$. In order to obtain sharper upper bounds, other linear constraints on the coefficents $a_{i}$ must be considered. The following theorem motivates the study of permutation arrays of given weight.

Theorem 7. Let $C$ be an $(n, d)-$ permutation code and $a_{i}=\frac{1}{|C|} \xi_{C}^{T} A_{i} \xi_{C}$. Let $D=$ $\left\{i_{1}, \ldots, i_{k}\right\}$ be the set of indices of the conjugacy classes whose elements have $n-w$ fixed points. Then

$$
\sum_{i \in D} a_{i} \leqslant \mu(n, d, w)
$$




\begin{tabular}{|c|c|c|}
\hline$\mu(n, d)$ & $n \mu(n-1, d)$ & Previous known bound \\
\hline$\mu(11,4)$ & 3326400 & 3628800 \\
$\mu(12,4)$ & 39916800 & 39916800 \\
$\mu(12,5)$ & 4354560 & 7149277 \\
$\mu(13,5)$ & 56609280 & 78823048 \\
$\mu(14,5)$ & 792529920 & 947590121 \\
$\mu(12,6)$ & 1663200 & 3926242 \\
$\mu(13,6)$ & 21621600 & 29511947 \\
$\mu(14,6)$ & 302702400 & 351525367 \\
$\mu(14,7)$ & 58287460 & 106314989 \\
$\mu(14,8)$ & 12312902 & 17297280 \\
\hline
\end{tabular}

Table 2: Upper bounds for $\mu(n, d)$ obtained by $\mu(n, d) \leqslant n \mu(n-1, d)$

Proof. For each $i, a_{i}|C|$ counts the number of pairs of permutations $(\phi, \psi)$ with $\phi, \psi \in C$ and $\phi \psi^{-1} \in C_{i}$, or, equivalently, the sum for $\phi \in C$ of the number of permutations $\psi \in C$ such that $\phi \psi^{-1} \in C_{i}$. The conjugacy classes are disjoint so we can write $|C| \sum_{i \in D} a_{i}=$ $\sum_{\phi \in C}\left|\left\{\psi \in C: \phi \psi^{-1} \in \cup_{i \in D} C_{i}\right\}\right|$. For each $\phi \in C$, the set $r_{\phi}\left(\left\{\psi \in C: \phi \psi^{-1} \in \cup_{i \in D} C_{i}\right\}\right)$ is composed of permutations of weight $w$, so $|C| \sum_{i \in D} a_{i}=\sum_{\phi \in C} \mu(n, d, w)$, and this concludes the proof.

Denote by $A(n, d, w)$ the maximum possible size of a constant weight $w$ binary code of length $n$ and distance $d$. Properties and known values of $A(n, d, w)$ for small values of the parameters can be found in [1]. In [17], Yang, Dong and Chen stated properties of $\mu(n, d, w)$ for $w \leqslant d$.

Theorem 8. Yang, Dong and Chen[17]

(i) $\mu(n, d, w) \leqslant A(n, 2 d-2 w, w) \quad$ for $w<d$

(ii) $\mu(n, d, w)=1 \quad$ for $2 w<d, w \neq 1$

(iii) $\mu(n, 2 k, k)=\left\lfloor\frac{n}{k}\right\rfloor \quad$ for $2 \leqslant k \leqslant\left\lfloor\frac{n}{2}\right\rfloor$

(iv) $\mu(n, 2 k+1, k+1)=A(n, 2 k, k+1) \quad$ for $1 \leqslant k \leqslant\left\lfloor\frac{n-1}{2}\right\rfloor$

(v) $\mu(n, 4,3) \leqslant \frac{n(n-1)}{3}$ for $n \geqslant 4$

The following theorem provides upper bounds for $\mu(n, d, w)$ even if $w>d$.

Theorem 9. For all $n \geqslant 3$, 
(i) $\mu(n, n, n)=n-1$

(ii) $\mu(n, n, n-1)=n$

(iii) $\mu(n, d, w) \leqslant\left(\begin{array}{l}n \\ k\end{array}\right) \mu(k, d, w) \quad$ for $w \leqslant k<n$

(iv) $\mu(n, d, w) \leqslant \mu(n-1, d, w)+(n-1)(\mu(n-1, d, w-1)+\mu(n-2, d, w-2)) \quad$ for $w<n$

(v) $\mu(n, d, n) \leqslant(n-1)(\mu(n-1, d, n-1)+\mu(n-2, d, n-2) \quad$ for $2 \leqslant d<n$

(vi) $\mu(n, n-2, n) \leqslant(n-1)(\mu(n-1, n-2)-1)$

Proof. The set consisting of the identity and all permutations of a $(n, n)$-code of weight $n$ is a $(n, n)$-code. Equality $(i)$ immediately follows from $\mu(n, n)=n$. In [7], G. Chang proved that a diagonal partial latin square whose entries are $1,2, \ldots, n$ can always be completed in a latin square, such a latin square corresponds to a $(n, n)$-code of weight $n-1$, and so (ii) holds.

If $C$ is a $(n, d)$-code of weight $w$, then for each $k$-subset $K$ of $\{1, \ldots, n\}$, the permutations $\phi \in C$ with $\operatorname{supp}(\phi) \subset K$ form a set isometric to a $(k, d)$-code of weight $w$. This leads to inequality $($ iii).

Denote by $C^{i}$ the subset of permutations $\phi$ in a $(n, d)$-code $C$ of weight $w$ such that $\phi(1)=i$. If $w<n$, the subset $C^{1}$ is a $(n-1, d)$-code of weight $w$. For $i=2, \ldots, n$, $l_{(1, i)}\left(C^{i}\right)$ consists of permutations whose support is of cardinality $w-1$ and of permutations fixing 1 and $i$, with support of cardinality $w-2$, and so $\left|l_{(1, i)}\left(C^{i}\right)\right| \leqslant \mu(n-1, d, w-1)+$ $\mu(n-2, d, w-2)$. Any $(n, d)$-code of weight $w$ can be written as a disjoint union $C=\cup_{i=1}^{n} C^{i}$, proving inequality $(i v)$. If $w=n$ then $C^{1}$ is empty, and the corresponding inequality is $(v)$. For $w=n$ and $d=n-2$, and for $i=2, \ldots, n$ each of subset $l_{(1, i)}\left(C^{i}\right)$ is isometric to a $(n-1, n-2)$-code whose all elements have support at least $n-2$. Such a code can be completed with the identity permutation and therefore has size less than $\mu(n-1, n-2)-1$, hence equality $(v i)$.

The upper bounds given in Theorem 9 are not sharp. For example, a clique search inspired by the method developped in [8] gives $\mu(6,5,5)=15$, while the upper bound obtained by application of Theorem is $9 \mu(6,5,5) \leqslant 34$. For this reason, the upper bounds do not contribute to any improvement of the results given by Theorem 7 for the range of values considered in Tables 1 and 2 .

\section{References}

[1] Erik Agrell, Alexander Vardy, and Kenneth Zeger. Upper bounds for constant-weight codes. IEEE Transactions on Information Theory, 46(7):2373-2395, 2000.

[2] V. B. Balakirsky and A. J. Han Vinck. On the performance of permutation codes for multi-user communication. Probl. Inf. Transm., 39(3):239-254, 2003. 
[3] Ian Blake. Permutation codes for discrete channels. IEEE Tansactions on Information Theory, pages 138-140, 1974.

[4] Mathieu Bogaerts. Codes et tableaux de permutations: construction, énumération et automorphismes. PhD thesis, Université Libre de Bruxelles, June 2009.

[5] Wieb Bosma, John J. Cannon, and Catherine Playoust. The Magma algebra system. I. The user language, 1997. http://magma .maths . usyd.edu.au/magma/.

[6] P. J. Cameron. Metric and geometric properties of sets of permutations. In Deza, Frankl, and Rosenberg, editors, Algebraic, Extremal and Metric Combinatorics 1986, pages 39-53. Cambridge University Press, 1988.

[7] Gerard J. Chang. Complete diagonals of latin square. Canadian Bulletin of Mathematics, 22(4):477-481, 1979.

[8] Wensong Chu, Charles J. Colbourn, and Peter Dukes. Construction for permutation codes in powerline communications. Des. Codes Cryptography, 32:51-64, 2004.

[9] P. Delsarte. An algebraic approach to the association schemes of coding theory. Technical report, Philips Research Reports, 1973.

[10] M. Deza and P. Frankl. On the maximum number of permutations with given maximal or minimal distance. Journal of Combinatorial Theory (A), 22:352-360, 1977.

[11] Michael Deza and Tayuan Huang. Metrics on permutations, a survey. J. Combinatorics, Information and System Sciences, 23:173-185, 1998.

[12] H. Farahat. The symmetric group as a metric space. Journal of London Mathematical Society, 35:215-220, 1960.

[13] The GAP Group. GAP - Groups, Algorithms, and Programming, Version 4.4.12, 2008. http://www. gap-system.org.

[14] MATLAB 7, 2004. http://www.mathworks.com/.

[15] Hannu Tarnanen. Upper bounds on permutation codes via linear programming. European J. Combinatorics, 20:101-114, 1999.

[16] A.J. Han Vinck. Coded modulation for power-line communications. AE Int. J. Electron. and Commun., 54(1):45-49, 2000.

[17] Lizhen Yang, Ling Dong, and Kefei Chen. New upper bounds on sizes of permutation arrays. CoRR, abs/0801.3983, 2008. http://arxiv.org/abs/0801.3983. 